\title{
Processo de Validação da Global Measure of Sexual Satisfaction em Três Amostras da População Portuguesa
}

\author{
Validation Process of the Global Measure of Sexual Satisfaction in Three Samples \\ of the Portuguese Population
}

\author{
Patrícia Monteiro Pascoal ${ }^{*}, b$, Isabel de Santa Bárbara Narciso ${ }^{a}$, \\ Nuno Monteiro Pereira ${ }^{b} \&$ Ana Sousa Ferreira ${ }^{a}$ \\ ${ }^{a}$ Universidade de Lisboa, Lisboa, Distrito de Lisboa, Portugal \\ $\&^{b}$ Associação para o Estudo Avançado da Sexualidade Humana, Lisboa, Distrito de Lisboa, Portugal
}

\begin{abstract}
Resumo
A satisfação sexual é um indicador importante da saúde sexual, contudo, a sua definição não é consensual. A Global Measure of Sexual Satisfaction (GMSEX) é um instrumento que avalia a satisfação sexual global no contexto de uma relação íntima. Pretendeu-se avaliar o comportamento psicométrico deste instrumento em três amostras distintas da população portuguesa, constituídas por participantes comprometidos em relações de coabitação: duas amostras presenciais designadas por "grupo normativo" sem diagnóstico e percepção de problemas sexuais $(n=291)$, "grupo clínico" com problemas sexuais $(n=84)$, e uma amostra online $(n=760)$. Confirmou-se a estrutura unifactorial da escala, verificando-se uma adequada fiabilidade e validade do instrumento.

Palavras-chave: Satisfação sexual, saúde sexual, coabitação, processo de validação.
\end{abstract}

\begin{abstract}
Sexual satisfaction is an important indicator of sexual health, however, its definition is not consensual. Lawrence and Byers (1995) developed a conceptual model of sexual satisfaction for people in committed relationships and, within this model, the authors developed the Global Measure of Sexual Satisfaction (GMSEX) which assesses global sexual satisfaction in the context of an intimate relationship. In the present study we aimed to adapt and assess the psychometric properties of this scale with three distinct samples from the Portuguese population in cohabitation relationships. One was designated as "normative group" with no diagnosis and perception of sexual problems ( $n=$ $291)$, another called "clinical group", whose participants presented sexual problems $(n=84)$, and an "online" sample of participants with and without perception of sexual problems $(n=760)$. The scale proved to have a single factor structure as well as good reliability and validity.

Keywords: Sexual satisfaction, sexual health, cohabitation, instrument validation.
\end{abstract}

A satisfação sexual é considerada um barómetro da qualidade da vida sexual (Sprecher, Christopher, Cate, Vangelisti, \& Perlman, 2006), sendo o seu estudo relevante dado que: (a) constitui um aspecto central da saúde sexual (Edwards \& Coleman, 2004), que é definida pela Organização Mundial de Saúde como um "estado de bem-estar físico, emocional e mental relacionado com a sexualidade" (World Health Organization, 2002); (b) tem sido, de forma consistente, associada ao bem-estar individual (Arrington,

" Endereço para correspondência: Faculdade de Psicologia, Universidade de Lisboa, Alameda da Universidade, Lisboa, Distrito de Lisboa, Portugal 1649-013. E-mail: patriciapascoal@campus.ul.pt

Este trabalho de investigação teve o apoio da Fundação para a Ciência e Tecnologia através da bolsa de doutoramento com a referência SFRH/BD/39934/2007.

Agradece-se à Marta Crawford o contributo na recolha de dados juntos da população clínica.
Cofrancesco, \& Wu, 2004; Heiman, LoPiccolo, \& Palladini, 1988), à qualidade conjugal (Byers, 2005; Sprecher et al., 2006; Young, Denny, Luquis, \& Young, 1998; Young, Denny, Young, \& Luquis, 2000) e à qualidade das relações amorosas (Althof et al., 2010; Byers, 2005; Byers, Wang, Harvey, Wenzel, \& Sprecher, 2004; Holmberg \& Blair, 2009; La France, 2010; Litzinger \& Gordon, 2005; Sprecher, 2002; Woloski-Wruble, Oliel, Leefsma, \& Hochner-Celnikier, 2010); (c) é uma das queixas comuns na população com problemas sexuais (Byers et al., 2004; DeLamater, Hyde, \& Fong, 2008; Sprecher, Cate, Harvey, \& Wenzel, 2004), especialmente na população feminina (Basson, 2003; Brotto et al., 2008; Hurlbert \& Apt, 1994; Leonard, Iverson, \& Follette, 2008; Meston \& Trapnell, 2005); (d) é considerado um objectivo central da actividade sexual de acordo com o modelo circular de resposta sexual humana desenhado especificamente para as mulheres (Basson, 2003). 
Apesar de ser um aspecto central na saúde sexual, a satisfação sexual tem sido definida de forma inconsistente, sem que haja um consenso quanto à sua operacionalização, o que tem uma tradução evidente na multiplicidade de instrumentos e metodologias de avaliação da satisfação sexual. As diferentes definições oscilam entre: (a) as que enfatizam a comparação entre um ideal (desejado) e um resultado concretizado (McClelland, 2010); (b) as que fazem corresponder a satisfação sexual à inexistência de problemas sexuais (Rust \& Golombok, 1985); (c) as que utilizam como indicadores a frequência da insatisfação e da discórdia sexual (Hudson, Harrison, \& Crosscup, 1981); (d) as que consideram a satisfação sexual como uma dimensão relacional do funcionamento sexual actual e passado (Wiegel, Meston, \& Rosen, 2005); (e) e as que acentuam a satisfação sexual como uma área da vida sexual traduzida pela avaliação subjectiva da experiência sexual individual no âmbito da relação actual (Byers \& Demmons, 1999; DeLamater et al., 2008; Lawrance \& Byers, 1995; MacNeil \& Byers, 2009), sendo definida por Lawrance e Byers (1995) como uma "resposta afectiva que decorre da avaliação subjectiva das dimensões positivas e negativas associadas com o seu relacionamento sexual" (p. 268).

Diferentes autores têm chamado a atenção para a necessidade de criar um método de avaliação da satisfação sexual que permita uma melhor e mais rigorosa comparabilidade e sistematização dos dados empíricos (McClelland, 2010; Stulhofer, Busko, \& Brouillard, 2010). Em consonância com as diferentes concepções de satisfação sexual, pode-se distinguir instrumentos que: (a) enfatizam a presença ou ausência de indicadores clínicos, como o Golombok Rust Inventory of Sexual Satisfaction (Rust \& Golombok, 1985, 1986); (b) avaliam dimensões relacionais, tais como proximidade relacional, a qualidade da comunicação, a satisfação na relação actual e satisfação global com a vida sexual, como o Female Sexual Functioning Index (Wiegel et al., 2005) ou o Index of Sexual Satisfaction (Hudson et al., 1981); (c) avaliam, de um modo integrativo, dimensões individuais e relacionais, tal como a New Sexual Satisfaction Scale (Stulhofer et al., 2010); (d) acentuam a avaliação subjectiva da componente interpessoal da sexualidade como a Global Measure of Sexual Satisfaction - GMSEX (Lawrance \& Byers, 1995); e (e) alguns instrumentos, como o The Pinney Sexual Satisfaction Inventory (Pinney, Gerrard, \& Denney, 1987), a Female Sexual Functioning Index (Wiegel et al., 2005) e a Sexual Satisfaction Scale for Women (Meston \& Trapnell, 2005), que contemplam as especificidades do género acerca da sexualidade.

As principais críticas aos instrumentos de avaliação, de um modo geral, salientam a sobreposição conceptual entre indicadores de funcionamento sexual (frequência, erecção, excitação, lubrificação, orgasmo) e indicadores de satisfação sexual; a confusão entre ausência de disfunção sexual e existência de satisfação sexual (McClelland, 2010); e a ausência de um modelo teórico que suporte as medidas existentes (Byers \& Macneil, 2006; Stulhofer et al., 2010).
Relativamente aos estudos de validação para a população portuguesa, a nossa revisão da literatura demonstrou que estão traduzidos, adaptados e com os respectivos estudos psicométricos publicados, o Female Sexual Function Index (Índice de Funcionamento Sexual Feminino) com uma amostra de mulheres com e sem problemas sexuais (Pechorro, Diniz, Almeida, \& Vieira, 2009), a New Sexual Satisfaction Scale (Escala de Satisfação Sexual; Refoios, Fuertes, \& Baz, 2011), e os estudos do Index of Sexual Satisfaction (Índice de Satisfação Sexual) com diferentes populações (Cardoso, 2003; Pechorro, Diniz, Almeida, \& Vieira, 2009).

No presente trabalho, pretende-se contribuir para o processo de validação da Global Measure of Sexual Satisfaction (GMSEX) na população portuguesa, a qual tem como suporte teórico o Modelo de Trocas Interpessoais da Satisfação Sexual (Interpersonal Exchange Modelo of Sexual Satisfaction [IEMSS]), desenvolvido por Lawrance e Byers (1995). O IEMSS deriva das teorias das trocas interpessoais que defendem que a apreciação global das relações humanas se baseia na avaliação dos aspectos negativos (custos) em relação aos positivos (recompensas). O IEMSS tem subjacente tais teorias na compreensão da satisfação sexual e constitui um modelo explicativo validado empiricamente da satisfação sexual. O GMSEX, desenvolvido no âmbito do IEMSS, constitui uma medida holística da satisfação sexual, estudada em relações amorosas diversificadas (Byers, 2005; Byers \& Demmons, 1999; Byers, Demmons, \& Lawrance, 1998; MacNeil \& Byers, 2009; Peck, Shaffer, \& Williamson, 2004), e revelando um comportamento psicométrico estável e consistente (Byers \& Macneil, 2006). Esta medida apresenta, ainda, sobre as restantes medidas supra-citadas, a vantagem de se adequar a ambos os sexos, ser de fácil compreensibilidade e rápida aplicação, não utilizar indicadores do funcionamento sexual, e ser uma medida específica da avaliação subjectiva da satisfação sexual. No presente trabalho, que pretende contribuir para a validação psicométrica da GMSEX junto da população portuguesa, foram realizados dois estudos com três amostras distintas, duas de recolha presencial (Estudo 1), e outra de recolha online (Estudo 2). Constituem, pois, objectivos deste trabalho: (a) analisar a validade de construto (factorial, convergente e divergente); (b) analisar a fiabilidade (consistência interna e correlação média inter-itens); (c) avaliar a sua capacidade discriminativa relativamente a participantes oriundos da amostra presencial clínica (com diagnóstico de problemas sexuais) e da amostra presencial normativa (sem diagnóstico e percepção de problemas sexuais), e relativamente a participantes com percepção subjectiva de problemas sexuais e participantes sem percepção de problemas sexuais, na amostra online.

\section{Método}

\section{Participantes}

Estudo 1. O Estudo 1 foi conduzido presencialmente com duas amostras de conveniência da população por- 
Pascoal, P. M. M., Narciso, I. S. B., Pereira, N. M. \& Ferreira, A. S. (2013). Processo de Validação da Global Measure of Sexual Satisfaction em Três Amostras da População Portuguesa.

tuguesa, comprometidos numa relação heterossexual de intimidade em situação de coabitação. A amostra normativa é constituída por participantes que, nas respostas ao protocolo de investigação aplicado, referiram não ter diagnóstico clínico conhecido e não revelaram auto-percepção de problema sexual. Esta amostra inclui 93 participantes (22 homens e 71 mulheres), sendo a idade média de 29,3 $(D P=10,5)$, variando entre os 18 e os 57 anos (Tabela 1$)$. Dada a prevalência de cerca de $90 \%$ de valores omissos, não foi calculada a média e desvio-padrão relativamente à duração da relação. A amostra clínica, com diagnóstico de problemas sexuais, inclui 84 participantes (45 homens e 39 mulheres), tem uma idade média de 36,59 anos ( $D P$ $=11,76)$, variando entre os 19 e os 73 anos. A duração média do casamento/união de facto é de 9,66 anos ( $D P$ $=10,82$ ). A maioria dos participantes tem formação universitária $(70,4 \%)$.

Estudo 2. Participaram 760 indivíduos (311 homens e 449 mulheres) da população portuguesa, oriundos de diferentes zonas do país, com idade média de 36,05 anos $(D P=8,34)$, variando entre os 20 e os 65 anos. A duração média do casamento/união de facto é de 7,4 anos $(D P=$ $7,35)$, variando entre 1 e 39 anos. A amostra é constituída por um subgrupo que, à semelhança de procedimentos utilizados noutros estudos para identificar a existência de problemas sexuais na população não clínica (McCabe \& Goldhammer, 2011), respondeu afirmativamente à questão: "Sente que tem algum problema sexual?", assinalando, posteriormente, numa tabela, quais os problemas específicos auto-identificados. Este grupo é designado como grupo com percepção subjectiva de problemas sexuais (91 participantes). A amostra é ainda constituída por um grupo sem percepção de problemas sexuais (669 participantes), constituído pelos participantes que referiram não ter nenhum problema sexual (Tabela 1). Para efeitos de comparação destes dois grupos (com e sem auto-percepção de problemas sexuais), e dada a diferença no número de participantes, efectuou-se uma extracção aleatória de cerca de $20 \%$ dos casos do grupo sem percepção de problemas sexuais, ficando, assim, composto por 146 participantes, com idade média de 36,24 anos $(D P=8,00)$, variando entre 20 e 65 anos; a duração média do casamento/união de facto é de $8,06(D P=7,17)$. No grupo com percepção subjectiva de problemas sexuais, a idade média dos participantes é de 36,4 anos $(D P=8,16)$, variando entre os 24 e os 62 anos; a duração média do casamento/união de facto é de 8,04 anos $(D P=7,01)$.

\section{Instrumentos (Estudo 1 e Estudo 2)}

Questionário Sócio-Demográfico. Foi construído um questionário para recolher dados sócio-demográficos dos participantes, tais como sexo, idade, habilitações literárias, estrutura relacional e orientação sexual (Tabela 1).

Global Measure of Sexual Satisfaction (GMSEX; Lawrance \& Byers, 1995). Avalia a satisfação sexual através da apreciação subjectiva que cada pessoa faz da sua relação sexual actual com o/a seu/sua companheiro/a através de cinco itens que adjectivam de forma bipolar a relação, numa escala de Likert de 7 pontos ("Muito Boa"-7 a 1 -"Muito Má"; "Muito Agradável" -7 a 1 -"Muito Desagradável"; "Muito Positiva" -7 a 1 - "Muito Negativa"; "Muito Satisfatória"-7 a 1 -“"Muito Insatisfatória"; "Muito Importante" -7 a 1 - "Muito Irrelevante"). Nos estudos originais, este instrumento demonstrou ter boa consistência interna com um alfa de Cronbach de 0,90 (Lawrance \& Byers, 1995). A fiabilidade temporal no estudo original foi estudada com intervalos de aplicação de 2 semanas e 3 meses, e apresentou índice de correlação de $r=0,84$ entre as duas primeiras aplicações, e $r=0,78(p<0,001)$ entre a segunda e terceira aplicação (Lawrance \& Byers, 1995). Nos estudos iniciais, observou-se, também, adequada validade convergente com o Index of Sexual Satisfaction (Hudson et al., 1981) com índice de correlação de $r=0,70,(p<$ 0,001; Byers et al., 1998; Lawrance \& Byers, 1995). Num estudo com uma amostra de 500 mulheres portuguesas, o valor da validade convergente com o inventário Golombok Rust Inventory of Sexual Satisfaction foi de $r=0,42,(p$ $<0,001)$. No âmbito do estudo preliminar, a esse estudo, que foi desenvolvido com 171 mulheres, a análise psicométrica da GMSEX revelou um alfa de Cronbach de 0,98, garantindo a consistência interna da medida. Verificou-se, neste estudo preliminar, que a fiabilidade temporal (com um intervalo de 3 semanas entre as duas administrações) se encontra assegurada, com um valor de correlação entre teste-reteste de $r=0,85$ ( $p<0,001$; Vilarinho, 2010). Num outro estudo português (Refoios et al., 2011) com uma amostra constituída por 316 sujeitos, a escala apresentou valores de alfa de Cronbach $=0,94$. Nesta amostra, ficou demonstrada a validade convergente com a New Sexual Satisfaction Scale (Stulhofer et al., 2010), sendo o valor de correlação de $r_{\mathrm{s}}=0,62(p<0,001)$.

Subescala da Sexualidade da Escala de Avaliação da Satisfação em Áreas da Vida Conjugal (EASAVIC; Narciso \& Costa, 1996). Para permitir o estudo da validade convergente, utilizou-se uma subescala da Escala de Avaliação da Satisfação em Áreas da Vida Conjugal, uma medida de auto-relato que avalia não só a satisfação conjugal global, mas também a satisfação específica em diferentes áreas da vida conjugal, entre as quais a sexualidade. $\mathrm{O}$ instrumento global apresenta 44 itens que avaliam a satisfação em diferentes áreas da vida conjugal, numa escala de Likert de 6 pontos, desde nada satisfeito a completamente satisfeito. Os 5 itens que constituem a subescala da sexualidade questionam o participante acerca da satisfação com: a frequência das relações sexuais; o desejo sexual do/a próprio/a; percepção de desejo do outro; qualidade da relação sexual; e prazer com a vida sexual. Num estudo desenvolvido com uma amostra de conveniência de 315 mulheres e 292 homens portugueses numa relação conjugal a subescala da sexualidade apresentou alfa de Cronbach de 0,93 (Narciso, Santos, \& Ribeiro, 2010). No presente trabalho, esta subescala apresenta alfa de Cronbach na amostra normativa de 0,96 e correlação média inter-itens (CMII) de 0,79; na amostra clínica, $\alpha$ 
Tabela 1

Caracterização Sócio-Demográfica das Amostras do Estudo 1 e 2

\begin{tabular}{|c|c|c|c|c|}
\hline & \multicolumn{2}{|c|}{ Estudo 1} & \multicolumn{2}{|c|}{ Estudo 2} \\
\hline & $\begin{array}{l}\text { Amostra normativa } \\
n=93(\%)\end{array}$ & $\begin{array}{l}\text { Amostra } \\
\text { clínica } \\
n=84(\%)\end{array}$ & $\begin{array}{l}\text { Subgrupo sem } \\
\text { problemas sexuais } \\
n=669(\%)\end{array}$ & $\begin{array}{l}\text { Subgrupo com } \\
\text { problemas sexuais } \\
n=91(\%)\end{array}$ \\
\hline \multicolumn{5}{|l|}{ Sexo } \\
\hline Masculino & $22(23,6)$ & $45(55,7)$ & $279(41,7)$ & $32(35,2)$ \\
\hline Feminino & $71(76,4)$ & $39(44,3)$ & $390(58,3)$ & $59(64,8)$ \\
\hline \multicolumn{5}{|l|}{ Habilitações Literárias } \\
\hline até 4 anos & $1(1,1)$ & $1(1,2)$ & - & - \\
\hline $5^{\circ}-6^{\circ}$ ano & $1(1,1)$ & $5(6)$ & $1(0,1)$ & - \\
\hline $7^{\circ}-9^{\circ}$ ano & $5(5,4)$ & $12(14,2)$ & $20(3,0)$ & $5(5,5)$ \\
\hline $10^{\circ}-12^{\circ}$ & $20(21,5)$ & $7(8,3)$ & $68(10,2)$ & $11(12,1)$ \\
\hline Frequência Universitária & $29(31,2)$ & $10(12)$ & $70(10,7)$ & $11(12,1)$ \\
\hline Ensino Superior & $36(38,7)$ & $49(58,4)$ & $510(76,2)$ & $64(70,3)$ \\
\hline \multicolumn{5}{|l|}{ Nível Sócio-Económico ${ }^{1}$} \\
\hline Baixo & $12(12,9)$ & $6(7,1)$ & - & - \\
\hline Médio & $56(60,2)$ & $29(34,5)$ & $184(24,2)$ & $184(24,2)$ \\
\hline Médio-Alto/Elevado & $25(26,9)$ & $49(58,4)$ & $563(73,9)$ & $563(73,9)$ \\
\hline \multicolumn{5}{|l|}{ Zona de Residência } \\
\hline Norte & $19(20,4)$ & $1(1,9)$ & $83(12,4)$ & $13(12,6)$ \\
\hline Centro & $30(32,3)$ & $3(3,6)$ & $122(18,2)$ & $15(18)$ \\
\hline Grande Lisboa & $34(36,6)$ & $70(83,3)$ & $387(57,8)$ & $51(57,6)$ \\
\hline Alentejo & $3(3,2)$ & $8(9,5)$ & $19(2,8)$ & $3(2,9)$ \\
\hline Outra (s) & $7(7,5)$ & $2(2,3)$ & $58(8,6)$ & $9(9,9)$ \\
\hline \multicolumn{5}{|l|}{ Situação Relacional } \\
\hline Casamento & $39(41,9)$ & $47(55,9)$ & $376(56,2)$ & $46(50,5)$ \\
\hline União de Facto/Coabitação & $4(4,3)$ & $35(44,1)$ & $293(43,8)$ & $45(49,5)$ \\
\hline Namoro & $29(31,2)$ & & & \\
\hline Não tem relação/Não se aplica & $19(20,4)$ & & & \\
\hline Outra situação & $2(2,2)$ & & & \\
\hline
\end{tabular}

$=0,88$ e $C M I I=0,56$ e, na amostra recolhida online,$\alpha=$ 0,91 e $C M I I=0,59$.

Body Appearance Cognitive Distraction Scale (BACDS; Dove \& Wiederman, 2000). Para permitir o estudo da validade discriminante, utilizou-se uma subescala de auto-relato da Cognitive Distraction Scale que avalia a distracção cognitiva durante a actividade sexual através do foco de atenção na aparência corporal. Seguimos uma das orientações referidas por Moreira (2004), escolhendo um instrumento que meça um construto relacionado com o construto em estudo (Meana \& Nunnink, 2006; Pujols, Meston, \& Seal, 2010). Espera-se que existam correlações baixas entre os dois instrumentos, indicando que existe relação mas também suficiente discriminação entre ambos os construtos. A BACDS não apresenta itens ou indicadores relacionados directamente com a satisfação sexual. Esta medida é composta por um conjunto de 10 itens, numa escala de Likert de 6 pontos. O coeficiente de consistência interna para a subescala de Aparência Corporal foi de 0,94 com uma amostra de homens e mulheres (Meana \& Nunnink, 2006). No presente trabalho, os valores encontrados de fiabilidade para a amostra normativa foram de $\alpha=0,89$ e $C M I I=0,47$; na amostra clínica, de $\alpha=0,84$ e $C M I I=$ $0,44 \mathrm{e}$, na amostra online, de $\alpha=0,92$ e $C M I I=0,58$.

\section{Procedimento}

A versão original do GMSEX foi submetida aos procedimentos habituais de tradução e retroversão, tendo sido desenvolvido em quatro passos: (a) tradução independente da versão original por dois especialistas na área e posterior elaboração de uma versão final; (b) retroversão para a língua inglesa por um psicólogo bilingue; (c) ajustes finais.
${ }^{1} \mathrm{O}$ nível sócio-económico foi identificado com base na profissão e nas habilitações dos participantes. Apesar de termos consciência de que, para um cálculo optimizado desta variável, teríamos de conhecer os rendimentos do próprio e do agregado familiar, entendemos que não haveria vantagens em obter tal informação, até porque poderia ser percepcionada como potencialmente intrusiva por parte de alguns respondentes. Demos maior relevo à vertente educacional, pois consideramos que esta caracteriza melhor o nível social dos participantes relativamente às oportunidades de acesso a informação adequada nas áreas em estudo neste trabalho. 
Pascoal, P. M. M., Narciso, I. S. B., Pereira, N. M. \& Ferreira, A. S. (2013). Processo de Validação da Global Measure of Sexual Satisfaction em Três Amostras da População Portuguesa.

Foram efectuadas duas aplicações- piloto com, respectivamente, 15 e 20 estudantes universitários para verificar a adequação e compreensão da linguagem.

No Estudo 1, relativamente à amostra presencial normativa, foram efectuadas aplicações colectivas $(n=57)$ em instituições de ensino universitário, escolas públicas e uma câmara municipal, e aplicações individuais $(n=$ 36). Os dados relativos à amostra presencial clínica foram recolhidos em contexto público $(n=35)$ e privado $(n=$ 49). No contexto público, fez-se o pedido de colaboração a uma consulta de sexologia clínica de um Hospital Psiquiátrico da zona de Lisboa, o qual foi apreciado pela Comissão de Ética e do Núcleo de Investigação Científica desta instituição que aprovou o estudo e os procedimentos de recolha de dados. A recolha de dados foi implementada após a consulta de triagem. Em contexto privado, os dados foram recolhidos no consultório de três terapeutas sexuais acreditadas pela Sociedade Portuguesa de Sexologia Clínica, e no âmbito da consulta de Medicina Sexual de dois especialistas nesta área. Em ambos os contextos, público e privado, os clínicos confirmavam a existência de problema sexual. A todos os participantes das amostras presenciais, era entregue um envelope com o protocolo, informações sobre o estudo e sobre os procedimentos de preenchimento e devolução e formulário de consentimento.

Para a recolha de dados do Estudo 2 (amostra online), foi utilizada uma plataforma $\mathrm{Web}$, a qual foi pré-testada com cerca de 30 participantes que avaliaram e comentaram a adequabilidade formal e de conteúdo do protocolo. Após ajustes finais, o estudo foi lançado publicamente, sendo prestada informação acerca dos objectivos e indicado o URL da página do consentimento informado que continha informações sobre objectivos, condições de participação no estudo (idade mínima de 18 anos e nacionalidade portuguesa), garantia de não registo de IP e segurança e actualização do servidor. A recolha de dados foi desenvolvida online durante 4 meses. Em ambos os estudos, eram garantidos a confidencialidade e anonimato, era fornecido o contacto da investigadora, bem como informações sobre serviços de apoio técnico especializado.

Análises Estatísticas. Tendo em conta os objectivos previamente descritos, apresentar-se-á o estudo da validade factorial, convergente, discriminante, estudo da capacidade discriminativa e estudo da fiabilidade do instrumento. As análises estatísticas foram conduzidas através da utilização do SPSS 19.0 (SPSS Inc, Chicago IL, USA), e foram seguidos os mesmos procedimentos estatísticos, quer no Estudo 1, quer no Estudo 2.

Os estudos sobre a estrutura factorial do GMSEX foram realizados através da Análise das Ordens (AO) que se baseia nos mesmos princípios que a Análise em Componentes Principais (ACP), utilizando a matriz de correlações de Spearman, adequando-se assim a escalas ordinais, como as do presente estudo. Apesar de constituir uma medida global que originalmente é constituída por somente um factor, pretendeu-se realizar uma análise factorial exploratória para verificar se todos os itens da
GMSEX apresentam um peso factorial que fundamente a sua inclusão na adaptação portuguesa do instrumento.

Para o estudo da validade discriminante, foi analisada a relação entre a GMSEX e a BACDS, e, para o estudo da validade convergente, foi analisada a relação entre a GMSEX e a subescala da sexualidade da EASAVIC. A distribuição do total da subescala da sexualidade da EASAVIC afasta-se da curva de Gauss, o que viola os pressupostos do teste de significância sobre o coeficiente de correlação $r$ de Pearson, pelo que se utilizou o coeficiente de correlação rho de Spearman e correspondente teste de significância para o estudo da relação entre o total da GMSEX e o total da subescala em estudo.

Foram utilizadas diferentes métodos estatísticos para estudar a capacidade discriminativa da GMSEX: (a) a comparação do resultado da GMSEX das amostras clínica e normativa, através do teste Mann-Whitney, utilizando um método de análise mais robusto uma vez que o pressuposto da normalidade da distribuição para aplicação de métodos paramétricos foi violado em todos os casos estudados no presente trabalho (i.e., o teste Kolmogorov-Smirnov revelou desvios à normalidade estatisticamente significativos, sendo a distribuição da variável claramente assimétrica pois o rácio entre o coeficiente de assimetria e o seu erro padrão cai fora do intervalo [-2 e 2]); (b) uma regressão logística binária que permite determinar, a partir do total da GMSEX, a probabilidade de detectar se uma pessoa pertence ao grupo clínico e determinar a sensibilidade e especificidade do instrumento; e (c) a acuidade da GMSEX como instrumento diagnóstico indicador da existência das dificuldades sexuais através do estudo da Receiver Operating Characteristics Curve (ROC Curve), metodologia também utilizada para determinar a existência do melhor ponto de corte ou ponto discriminativo (cut off point) para que o instrumento seja usado para diferenciar a população clínica da não-clínica.

Tendo em conta que a regressão logística com apenas uma variável preditora dificilmente conduz a um bom ajustamento, completámos este estudo com a análise da ROC Curve. Para determinar o melhor valor de corte para a utilização do instrumento como diagnóstico, procedeu-se ao estudo com o total dos itens da GMSEX através ROC Curve. Esta curva posiciona a sensibilidade (que detecta os verdadeiros positivos) e a especificidade (que nos dá a taxa de falsos positivos) para cada resultado nos questionários. A área abaixo da curva (area under the curve-AUC) é um indicador da capacidade de distinguir entre "casos" (com a presença da característica em estudo) e "não casos" (sem a característica em estudo). O índice da AUC pode variar entre 0,5 , que indica uma possibilidade aleatória de associação, ou seja, nenhum valor diagnóstico, e 1,0, que indica perfeita discriminação entre "casos" e "não casos". Hosmer e Lemeshow (2000, citado em Maroco, 2007) apresentam valores indicativos de discriminação fraca (entre 0,5 e 0,7 ) e discriminação aceitável $(0,7$ a 0,8$)$, sendo um resultado superior a 0,8 considerado suficientemente discriminatório para que um instrumento possa servir de 
screening e diferenciar sujeitos com e sem a característica clínica em estudo. A determinação de um ponto de corte óptimo depende do equilíbrio entre a sensibilidade (capacidade de detectar positivos verdadeiros) e a especificidade (capacidade de determinar falsos positivos). Sensibilidade mais alta resulta em menos falsos negativos e a especificidade mais alta resulta em menos falsos positivos (casos erradamente diagnosticados). Os pontos de corte óptimos relacionam-se com os objectivos para que são utilizados e devem ter em conta o equilíbrio entre a sensibilidade e especificidade.

Para o estudo da capacidade discriminativa do instrumento com a amostra online $(n=760)$, como referido anteriormente, os estudos comparativos entre estes dois grupos foram efectuados após a extracção aleatória de uma sub-amostra sem dificuldades sexuais $(n=146)$ para que os grupos tivessem uma distribuição de casos mais aproximada. Para o estudo da fiabilidade, consideraram-se os valores do alfa de Cronbach e da CMII.

\section{Resultados}

Estudo de Validade

\section{Validade Factorial}

Estudo 1 - Amostra Presencial. Na Tabela 2, encontram-se descritos os resultados relativos ao estudo de validade factorial da GMSEX através da Análise das Ordens
(AO). De acordo com os resultados expressos na Tabela 2 , na amostra presencial normativa seriam seleccionados dois factores, quer pelo critério de Kaiser (valores próprios superiores a um) quer pelo critério da percentagem total de variabilidade explicada adequada (superior a 75\%). O $1^{\circ}$ factor está associado aos itens 2, 3 e 4 (Muito Agradável - Muito desagradável; Muito Positiva - Muito Negativa; Muito Satisfatória - Muito Insatisfatória) e o $2^{\circ}$ factor definido pelos itens 1 e 5 (Muito Boa - Muito Má; Muito Importante - Muito Irrelevante). Estes dois factores apresentam uma relação direta, moderada e significativa $\left(r_{\mathrm{s}}=0,25, p<0,001\right)$. Não se encontraram diferenças conceptuais que expliquem a existência de dois factores. Em coerência com o facto da GMSEX ser uma medida global da satisfação sexual, e, ainda, com a finalidade de manter a consistência teórica e conceptual deste estudo e viabilizar a comparação dos resultados com estudos prévios, optou-se por considerar um único factor com a totalidade dos itens nas análises subsequentes. $\mathrm{Na}$ amostra presencial clínica (ver Tabela 2), foi seleccionado um factor, quer pelo critério de Kaiser (valores próprios superiores a um), quer pelo critério da percentagem total de variabilidade explicada adequada (superior a $75 \%$ ).

Estudo 2 -Amostra Online. Os resultados confirmam a estrutura unifactorial da GMSEX (ver Tabela 2), quer pelo critério de Kaiser (valores próprios superiores a um), quer pelo critério da percentagem total de variabilidade explicada adequada (superior a $75 \%$ ).

Tabela 2

Estrutura Factorial Final da GMSEX (Análise das Ordens) na Amostra Presencial Normativa ( $n=93$ ), na Amostra Presencial Clínica $(n=84)$ e na Amostra Online $(n=760)$

\begin{tabular}{|c|c|c|c|c|}
\hline & \multicolumn{3}{|c|}{ Estudo 1} & \multirow{2}{*}{$\begin{array}{c}\text { Estudo } 2 \\
\text { Amostra Online }\end{array}$} \\
\hline & Amostra Pr & Normativa & Amostra Presencial Clínica & \\
\hline Itens & Factor 1 & Factor 2 & Factor 1 & Factor 1 \\
\hline 1 & 0,087 & $\mathbf{0 , 8 5 5}$ & 0,942 & 0,943 \\
\hline 2 & 0,988 & $-0,059$ & 0,965 & 0,938 \\
\hline 3 & 0,987 & 0,093 & 0,960 & 0,936 \\
\hline 4 & 0,980 & $-0,075$ & 0,942 & 0,925 \\
\hline 5 & 0,178 & 0,837 & 0,659 & 0,728 \\
\hline $\begin{array}{c}\text { Percentagem de } \\
\text { variabilidade explicada }\end{array}$ & 58,98 & 28,97 & 81,22 & 80,26 \\
\hline $\begin{array}{l}\text { Percentagem de variabilidade } \\
\text { explicada acumulada }\end{array}$ & & & & \\
\hline
\end{tabular}

Validade Convergente. No Estudo 1, o resultado da correlação entre a GMSEX e a sub-escala de Sexualidade da EASAVIC na amostra presencial normativa foi de $r_{\mathrm{s}}$ $=0,50(p=0,003)$ e, na amostra presencial clínica, foi de $r_{\mathrm{s}}=0,62(p<0,001)$, o que revela uma relação directa, moderada e significativa, evidenciando que, quanto mais elevados os valores indicadores de satisfação sexual num dos instrumentos, maiores os valores indicadores de satisfação sexual no outro instrumento. Relativamente ao Estudo 2, com a amostra recolhida online, o valor do 
Pascoal, P. M. M., Narciso, I. S. B., Pereira, N. M. \& Ferreira, A. S. (2013). Processo de Validação da Global Measure of Sexual Satisfaction em Três Amostras da População Portuguesa.

coeficiente de correlação entre a GMSEX e a sub-escala de Sexualidade da EASAVIC, na amostra online, foi de $r_{\mathrm{s}}=0,82(p<0,001)$. O valor encontrado, é indicador de uma relação directa, muito forte e significativa.

Validade Discriminante. No Estudo 1, o resultado da correlação entre a GMSEX e a BACDS na amostra normativa foi de $r_{\mathrm{s}}=-0,37(p<0,05)$, um valor estatisticamente significativo que indica haver uma relação inversa, moderada e significativa entre as duas medidas. Na amostra clínica, o valor $r_{\mathrm{s}}=-0,16(p=0,176)$ revela uma relação inversa, fraca e não significativa entre as duas variáveis. No Estudo 2, o resultado da correlação entre a GMSEX e a BACDS foi de $r_{\mathrm{s}}=-0,26(p<0,01)$, um valor indicativo de uma relação inversa, fraca e significativa entre os dois instrumentos.

Capacidade Discriminativa. No Estudo 1, a média do total da GMSEX, na amostra normativa $(M=25,64, D P$ $=7,57, n=93)$, é superior à média na amostra clínica $(M$ $=21,22, D P=6,86, n=84)$ e a utilização do teste $U$ de Mann-Whitney revelou que a distribuição dos níveis de satisfação sexual tem valores superiores na amostra normativa $(z=-3,599$, valor $p / 2=0,001$, magnitude do efeito $=-0,256$ ), indicando uma satisfação sexual mais elevada.

Foi efectuada uma regressão logística binária, tendo como variável dependente a pertença $(n=84)$ ou não pertença $(n=93)$ à amostra clínica. O modelo de regressão mostrou-se significativo $\left[\chi^{2}(1)=19,895, p<0,001\right]$, apresentando uma percentagem de bem classificados de $68,2 \%$, estimada por re-substituição. Este valor é superior à percentagem de bem classificados pela regra da maioria $(57,4 \%)$. A especificidade foi de $90 \%$ e a sensibilidade de $28,6 \%$, sendo, este último, um valor considerado baixo e que parece indicar que este instrumento é pouco sensível para diferenciar a população clínica da não clínica. O teste de Hosmer e Lemeshow revelou resultados significativos $(\chi 2=32,321, p<0,001)$. Para que se possa considerar que o modelo é bem ajustado, não se deve rejeitar a hipótese nula do teste de Hosmer e Lemeshow, pelo que, no presente trabalho, os valores encontrados não conduzem a um bom ajustamento do modelo de regressão.

No presente estudo, a Curva ROC apresentou uma área abaixo da curva de 0,680 $(p<0,001$ e 95\% IC: 0,601-0,760), um valor indicador de discriminação fraca pelo que não foi feito o estudo do ponto de corte óptimo. Quanto à validade discriminativa no Estudo 2, a média do total da GMSEX no grupo que auto-percepciona não ter um problema sexual é mais elevada $(M=29,44, D P$ $=5,6, n=146)$ do que no grupo que auto-percepciona a existência de um problema sexual $(M=23,57, D P=$ $6,33, n=91$ ). No Estudo 2, o teste $U$ de Mann-Whitney revelou que a distribuição dos níveis de satisfação sexual tem valores superiores no grupo que auto-percepciona não ter um problema sexual $(z=-6,825$, valor $p / 2=0,001$, magnitude do efeito $=-0,44)$ indicando que a satisfação sexual é mais elevada. Foi realizada uma regressão logística binária, tendo como variável dependente a percepção de ter ou não problemas sexuais. Foram introduzidos, na análise, um total de 237 casos, sendo 91 pertencentes ao grupo com percepção subjectiva de problema sexual. A inclusão do valor do total da GMSEX produziu um modelo significativo $\left[\chi^{2}(1)=47,591, p<0,001, n=237\right]$ com uma variância explicada entre $18,2 \%$ ( $R$ quadrado de Cox e Snell) e $24,7 \%$ ( $R$ quadrado Nagelkerke) e uma percentagem de casos bem classificados de $70 \%$, estimada por re-substituição. $\mathrm{O}$ valor determinado para a sensibilidade apresentada é de $51,6 \%$ e a especificidade de $81,5 \%$. A análise da ROC Curve determinou uma área abaixo da curva de $0,761(p<0,001,95 \%$ IC: $0,712-0,809)$ um valor indicativo de poder discriminativo aceitável. Não foi feita a determinação do melhor ponto de corte uma vez que valores de AUC inferiores ou aproximados de 0,75 não são considerados clinicamente úteis (Fan, Upadhye, \& Worster, 2006).

Estudo de Fiabilidade. Relativamente ao Estudo 1, a avaliação da fiabilidade, que considerou os valores do alfa de Cronbach e de CMII, revelou bons níveis de fiabilidade, quer na amostra normativa $(n=291)$ com alfa de Cronbach $=0, .83$ e $C M I I=0,45$, quer na amostra clínica $(n=87)$, com alfa de Cronbach $=0,91$ e $C M I I=0,64$. No Estudo2, na amostra online ( $n=760)$, o alfa de Cronbach apresenta um valor de 0,94 e $C M I I=0,77$.

\section{Discussão}

O presente trabalho pretendeu estudar as qualidades psicométricas da versão portuguesa da Global Measure of Sexual Satisfaction (GMSEX), um instrumento destinado à avaliação da satisfação sexual global, conceptualizada como a valoração subjectiva da experiência sexual na relação amorosa actual. $\mathrm{O}$ trabalho foi desenvolvido junto de três amostras distintas da população portuguesa: (a) uma amostra presencial normativa sem auto-percepção nem diagnóstico de problemas sexuais; (b) uma amostra presencial clínica com diagnóstico de problemas sexuais; e (c) uma amostra recolhida online de participantes com e sem percepção de problemas sexuais.

Iniciou-se o estudo com a análise factorial do instrumento através da Análise das Ordens (AO). A estrutura unifactorial original foi replicada na amostra presencial clínica e na amostra online, contudo, na amostra presencial normativa, a análise factorial resultou numa estrutura bifactorial. Através da análise do conteúdo dos itens constituintes dos dois factores encontrados através da $\mathrm{AO}$, não se encontraram diferenças semânticas que distingam um factor do outro, apesar da baixa correlação encontrada entre estes indicar que os factores não estão fortemente associados. Julgamos pertinente considerar que a emergência deste $2^{\circ}$ factor na amostra presencial normativa pode estar relacionada com o facto de esta amostra ser constituída exclusivamente por participantes sem relato de diagnóstico clínico ou de auto-percepção de problemas sexuais. Tal poderá significar que, quando se verificam ou se auto-percepcionam problemas sexuais, a auto-avaliação relativa à satisfação sexual é mais holística; 
quando não se verificam ou não se auto-percepcionam problemas sexuais, eventualmente, os sujeitos são mais analíticos, o que poderá levar a uma diferenciação mais fina da semântica inerente aos vários itens. No entanto, seriam necessários estudos específicos que investigassem esta hipótese. Acresce, ainda, como possível explicação para a emergência do $2^{\circ}$ factor na amostra presencial normativa, o facto de ser uma amostra mais heterogénea em termos de literacia, situação relacional e características sócio-demográficas (Byers et al., 1998; Byers \& Macneil, 2006; Lawrance \& Byers, 1995; Renaud, Byers, \& Pan, 1997). Assim, optou-se por manter a estrutura encontrada na escala original.

A validade de constructo do instrumento foi também estudada através do coeficiente de correlação rho de Spearman entre a escala global e a subescala da Sexualidade da EASAVIC, que também avalia a satisfação com a sexualidade (validade convergente). Estudou-se, ainda, a relação entre a escala global e a BACDS, recorrendo ao coeficiente de correlação rho de Spearman. Relativamente ao estudo da validade convergente, verificou-se existir uma relação moderada significativa na amostra presencial normativa, e relações diretas fortes entre os instrumentos em estudo na amostra presencial clínica e na amostra online. A relação encontrada com a amostra normativa foi mais fraca que as demais relações, o que pode ser explicado pela junção dos 2 fatores indicados pela Análise das Ordens. As relações encontradas entre a GMSEX e a subescala da Sexualidade da EASAVIC fazem sentido, pois apesar de utilizarem indicadores e pontos de partida teóricos diferentes, ambos os instrumentos avaliam a satisfação sexual. Assim, os resultados encontrados suportam, em termos estatísticos, a validade convergente do instrumento.

Relativamente ao estudo da validade divergente esperava-se que, os resultados suportassem que, apesar de relacionados, os instrumentos são distintos, não existindo uma relação moderada ou forte entre eles. As relações encontradas com a BACDS foram inversas, o que sugere que quanto maior o grau de distracção negativa, menor a satisfação sexual. A relação encontrada, embora significativa, foi moderada (com um valor próximo de relação fraca), na amostra presencial normativa, e fraca na amostra online. Na amostra presencial clínica, não se encontrou relação estatisticamente significativa entre os dois instrumentos. Globalmente, os constructos estão fracamente relacionados e são distintos. Os resultados do estudo da relação da GMSEX com a BACDS suportam a existência de validade divergente, sendo que os resultados encontrados na população normativa presencial não são inequívocos. Esta amostra difere em algumas variáveis sócio-demográficas das restantes amostras em estudo, pelo que os resultados podem ser explicados pela não existência de equivalência sócio-demográfica entre as amostra e pelo facto desta amostra só incluir participantes sem problemas sexuais . Esta diferença pode, ainda, ser explicada pela opção, baseada em critérios teóricos, de agrupar num só factor os dois factores que resultaram do estudo da AO. Estudos futuros devem ter este aspecto em conta de forma a esclarecer a natureza da associação entre os dois constructos.

No Estudo 1, a avaliação da capacidade discriminativa do instrumento foi efectuada através da comparação das médias totais do instrumento entre a amostra normativa e a amostra clínica. Utilizou-se o teste $U$ de Mann-Whitney, e os resultados encontrados demonstram que há diferenças significativas entre as duas amostras. Também no Estudo 2 , na amostra online, os resultados do teste $U$ de Mann-Whitney relativamente à comparação das médias totais do grupo com percepção de problemas sexuais e do grupo sem percepção de problemas sexuais revelam diferenças estatisticamente significativas. Utilizou-se a regressão logística binária nas amostras presenciais e online com o objectivo de estudar se o instrumento tem a capacidade de predizer a que grupo (clínico/não clínico, nas amostras presenciais; com auto-percepção/sem auto-percepção de problemas sexuais, na amostra online) pertence cada participante. Em todas as amostras (presenciais e online), o resultado da regressão logística binária confirma que o instrumento revela capacidade para classificar correctamente os participantes. Contudo, nas amostras presenciais, a sensibilidade do instrumento é baixa $(28,6 \%)$, parecendo indicar que este instrumento é pouco sensível para diferenciar a população clínica da não clínica. Já na amostra online, a sensibilidade é aceitável (51,6\%). Os valores de área debaixo da curva determinados a partir do estudo da Roc Curve confirmam a capacidade discriminativa fraca (amostras presenciais) e moderada (amostra online) do instrumento que acaba por se traduzir na falta de acuidade do mesmo para estabelecer um ponto de corte óptimo que permita diferenciar a população com problemas da população sem problemas. Estes resultados parecem indicar que, embora a GMSEX revele sensibilidade baixa para discriminar entre situações com e sem diagnóstico de dificuldades sexuais, permite, no entanto, diferenciar entre situações de percepção subjectiva de problemas sexuais e de percepção subjectiva de ausência de problemas.

Em termos de fiabilidade, a versão portuguesa do GMSEX revelou-se uma medida consistente com valores de alfa de Cronbach elevados. Os valores de CMII, que corresponde à média das correlações entre cada par de itens, são elevados e afastam-se do desejável, pois indicam homogeneidade nos itens de que é composta a escala. Este resultado, que nos indica que os itens que constituem a escala são, em média, semelhantes entre si, é consistente com as características da escala, uma vez que para cada item, se solicita repetidamente que o participante se posicione num extremo do continuum em relação à dimensão em estudo.

Em suma, considera-se que o instrumento pode, com cautela, ser utilizado em contexto de investigação específica com amostras clínicas pois não permite com clareza diferenciar pessoas com e sem diagnóstico de disfunção sexual, o que pode ser explicado pelo facto de satisfação 
Pascoal, P. M. M., Narciso, I. S. B., Pereira, N. M. \& Ferreira, A. S. (2013). Processo de Validação da Global Measure of Sexual Satisfaction em Três Amostras da População Portuguesa.

não significar necessariamente ausência de disfunção sexual, e insatisfação não significar necessariamente disfunção sexual (Althof et al., 2010; Dundon \& Rellini, 2010; Evangelia et al., 2010; Stephenson \& Meston, 2010). No entanto, considera-se que o instrumento pode e deve ser utilizado em complemento de instrumentos que avaliam o funcionamento sexual, pois permite ter uma visão mais ampla da saúde sexual. A validação para a população portuguesa da GMSEX é importante, sobretudo para a aplicação em contextos de investigação e clínico, dada a escassez de instrumentos validados em língua portuguesa; a escassez de instrumentos que tenham aplicabilidade em ambos os sexos; a sua fácil compreensão e rapidez de resposta; e a validade convergente encontrada com outras medidas que avaliam a satisfação sexual a partir de outros indicadores.

Estudos futuros deverão contemplar amostras mais amplas e diversificadas que permitam perceber se os resultados encontrados na nossa amostra presencial normativa se repetem em amostras com diferentes características relacionais. Seria, ainda, interessante, em estudos futuros, verificar se há especificidades na relação da satisfação sexual com os diferentes quadros clínicos, e se o instrumento revela maior capacidade discriminativa em amostras da população cuja principal queixa sexual seja a insatisfação sexual.

\section{Referências}

Althof, S. E., Buvat, J., Gutkin, S. W., Belger, M., Stothard, D. R., \& Fugl-Meyer, A. R. (2010). Sexual satisfaction in men with erectile dysfunction: Correlates and potential predictors. Journal of Sexual Medicine, 7(1), 203-215.

Arrington, R., Cofrancesco, J., \& Wu, A. W. (2004). Questionnaires to measure sexual quality of life. Quality of Life Research, 13(10), 1643-1658.

Basson, R. (2003). Biopsychosocial models of women's sexual response: Applications to management of 'desire disorders'. Sexual \& Relationship Therapy, 18(1), 107.

Brotto, L. A., Heiman, J. R., Goff, B., Greer, B., Lentz, G. M., Swisher, E., Van Blaricom, A. (2008). A psychoeducational intervention for sexual dysfunction in women with gynecologic cancer. Archives of Sexual Behavior, 37(2), 317-329.

Byers, E. S. (2005). Relationship satisfaction and sexual satisfaction: A longitudinal study of individuals in long-term relationships. Journal of Sex Research, 42(2), 113-118.

Byers, E. S., \& Demmons, S. (1999). Sexual satisfaction and sexual self-disclosure within dating relationships. Journal of Sex Research, 36(2), 180-189.

Byers, E. S., Demmons, S., \& Lawrance, K. A. (1998). Sexual satisfaction within dating relationships: A test of the interpersonal exchange model of sexual satisfaction. Journal of Social and Personal Relationships, 15(2), 257-267.

Byers, E. S., \& Macneil, S. (2006). Further validation of the interpersonal exchange model of sexual satisfaction. Journal of Sex \& Marital Therapy, 32(1), 53-69.

Byers, E. S., Wang, A., Harvey, J. H., Wenzel, A., \& Sprecher, S. (2004). Understanding sexuality in close relationships from the social exchange perspective. In The handbook of sexuality in close relationships (pp. 203-234). Mahwah, NJ: Lawrence Erlbaum.
Cardoso, J. (2003). Sexualidade masculina pós-lesão vertebro-medular (Tese de doutorado, Instituto de Ciências Biomédicas de Abel Salazar, Universidade do Porto, Portugal).

DeLamater, J., Hyde, J. S., \& Fong, M.-C. (2008). Sexual satisfaction in the seventh decade of life. Journal of Sex \& Marital Therapy, 34(5), 439-454.

Dove, N. L., \& Wiederman, M. W. (2000). Cognitive distraction and women's sexual functioning. Journal of Sex and Marital Therapy, 26, 67-78.

Dundon, C. M., \& Rellini, A. H. (2010). More than sexual function: Predictors of sexual satisfaction in a sample of women age 40-70. Journal of Sexual Medicine, 7(2), 896-904.

Edwards, W., \& Coleman, E. (2004). Defining sexual health: A descriptive overview. Archives of Sexual Behavior, 33(3), 189-195.

Evangelia, N., Kirana, P.-S., Chiu, G., Link, C., Rosen, R., \& Hatzichristou, D. (2010). Level of bother and treatmentseeking predictors among male and female in-patients with sexual problems: A hospital-based study. Journal of Sexual Medicine, 7(2), 700-711.

Fan, J., Upadhye, S., \& Worster, A. (2006). Understanding receiver operating characteristic (ROC) curves. CJEM: $\mathrm{Ca}$ nadian Journal of Emergency Medicine, 8(1), 19-20.

Heiman, J. R., LoPiccolo, J., \& Palladini, D. (1988). Becoming orgasmic: A sexual and personal growth program for women (Rev. Ed. Exp.). Paramus, NJ: Prentice Hall Press.

Holmberg, D., \& Blair, K. L. (2009). Sexual desire, communication, satisfaction, and preferences of men and women in same-sex versus mixed-sex relationships. Journal of Sex Research, 46(1), 57-66.

Hudson, W. W., Harrison, D. F., \& Crosscup, P. C. (1981). A short-form scale to measure sexual discord in dyadic relationships. Journal of Sex Research, 17(2), 157.

Hurlbert, D. F., \& Apt, C. (1994). Female sexual desire, response, and behavior. Behavior Modification, 18(4), 488-504.

La France, B. H. (2010). Predicting sexual satisfaction in interpersonal relationships. Southern Communication Journal, 75(3), 195-214.

Lawrance, K.-A., \& Byers, E. S. (1995). Sexual satisfaction in long-term heterosexual relationships: The interpersonal exchange model of sexual satisfaction. Personal Relationships, 2(4), 267-285.

Leonard, L. M., Iverson, K. M., \& Follette, V. M. (2008). Sexual functioning and sexual satisfaction among women who report a history of childhood and/or adolescent sexual abuse. Journal of Sex \& Marital Therapy, 34(5), 375-384.

Litzinger, S., \& Gordon, K. C. (2005). Exploring relationships among communication, sexual satisfaction, and marital satisfaction. Journal of Sex \& Marital Therapy, 31(5), 409-424.

MacNeil, S., \& Byers, E. S. (2009). Role of sexual self-disclosure in the sexual satisfaction of long-term heterosexual couples. Journal of Sex Research, 46(1), 3-14.

Maroco, J. (2007). Análise estatística com utilização do SPSS. Lisboa, Portugal: Sílabo.

McCabe, M. P., \& Goldhammer, D. L. (2011). Demographic and psychological factors related to sexual desire among heterosexual women in a relationship. Journal of Sex Research, 49(1), 78-87.

McClelland, S. I. (2010). Intimate justice: A critical analysis of sexual satisfaction. Social and Personality Psychology Compass, 4(9), 663-680.

Meana, M., \& Nunnink, S. E. (2006). Gender differences in the content of cognitive distraction during sex. Journal of Sex Research, 43(1), 59-67. 
Meston, C., \& Trapnell, P. (2005). Development and validation of a five-factor sexual satisfaction and distress scale for women: The sexual satisfaction scale for women (SSS-W). Journal of Sexual Medicine, 2(1), 66-81.

Moreira, J. (2004). Questionários: Teoria e prática. Coimbra, Portugal: Almedina.

Narciso, I., \& Costa, M. E. (1996). Amores satisfeitos, mas não perfeitos. Cadernos de Consulta Psicológica, 12, 115-130.

Narciso, I., Santos, S. V., \& Ribeiro, M. T. (2010). Satisfação conjugal e percepções retrospectivas do clima relacional na família de origem. Trabalho apresentado no VII Simpósio Nacional de Investigação em Psicologia, Braga, Portugal.

Pechorro, P., Diniz, A., Almeida, S., \& Vieira, R. (2009). Validação de uma versão feminina do índice de satisfação sexual (ISS). Laboratório de Psicologia, 7(1), 45-56.

Peck, S. R., Shaffer, D. R., \& Williamson, G. M. (2004). Sexual satisfaction and relationship satisfaction in dating couples: The contributions of relationship communality and favorability of sexual exchanges. Journal of Psychology \& Human Sexuality, 16(4), 17-37.

Pinney, E. M., Gerrard, M., \& Denney, N. W. (1987). The Pinney sexual satisfaction inventory. Journal of Sex Research, 23(2), 233.

Pujols, Y., Meston, C. M., \& Seal, B. N. (2010). The association between sexual satisfaction and body image in women. Journal of Sexual Medicine, 7(2), 905-916.

Refoios, S. M., Fuertes, A. M., \& Baz, B. O. (2011). Vinculos afectivos, comunicación sexual y satisfacción sexual en una muestra de parejas heterosexuales portuguesas. Salamanca, Espanha: Facultad de Psicología, Universidad de Salamanca.

Renaud, C., Byers, E. S., \& Pan, S. (1997). Sexual and relationship satisfaction in mainland China. Journal of Sex Research, 34(4), 399-410.

Rust, J., \& Golombok, S. (1985). The Golombok-Rust inventory of sexual satisfaction (GRISS). British Journal of Clinical Psychology, 24(1), 63-64.

Rust, J., \& Golombok, S. (1986). The griss: A psychometric instrument for the assessment of sexual dysfunction. Archives of Sexual Behavior, 15(2), 157-165.

Sprecher, S. (2002). Sexual satisfaction in premarital relationships: Associations with satisfaction, love, commitment, and stability. Journal of Sex Research, 39(3), 190.

Sprecher, S., Cate, R. M., Harvey, J. H., \& Wenzel, A. (2004). Sexual satisfaction and sexual expression as predictors of relationship satisfaction and stability. In J. Harvey, A. Wenzel, \& S. Sprecher (Eds.), The handbook of sexuality in close relationships (pp. 235-256). Mahwah, NJ: Lawrence Erlbaum.

Sprecher, S., Christopher, F. S., Cate, R., Vangelisti, A. L., \& Perlman, D. (2006). Sexuality in close relationships. In The cambridge handbook of personal relationships (pp. 463-482). New York: Cambridge University Press.

Stephenson, K. R., \& Meston, C. M. (2010). Differentiating components of sexual well-being in women: Are sexual satisfaction and sexual distress independent constructs? Journal of Sexual Medicine, 7(7), 2458-2468.

Stulhofer, A., Busko, V., \& Brouillard, P. (2010). Development and bicultural validation of the new sexual satisfaction scale. Journal of Sex Research, 47(4), 257-268.

Vilarinho, S. (2010). Funcionamento e satisfação sexual feminina: Integração do afecto, variáveis cognitivas e relacionais, aspectos biológicos e contextuais. Tese de Doutoramento: https://eg.sib.uc.pt/bitstream/10316/18484/1/TESE.pd, Universidade de Coimbra, Coimbra, Portugal.
Wiegel, M., Meston, C., \& Rosen, R. (2005). The female sexual function index (FSFI): Cross-validation and development of clinical cutoff scores. Journal of Sex \& Marital Therapy, 31(1), 1-20.

Woloski-Wruble, A. C., Oliel, Y., Leefsma, M., \& HochnerCelnikier, D. (2010). Sexual activities, sexual and life satisfaction, and successful aging in women. Journal of Sexual Medicine, 7(7), 2401-2410.

World Health Organization. (2002). Gender and reproductive rights, glossary, sexual health. Retrieved April 18, 2011, from http://www.who.int/topics/sexual_health/en/

Young, M., Denny, G., Luquis, R., \& Young, T. (1998). Correlates of sexual satisfaction in marriage. Canadian Journal of Human Sexuality, 7(2), 115-127.

Young, M., Denny, G., Young, T., \& Luquis, R. (2000). Sexual satisfaction among married women. American Journal of Health Studies, 16(2), 73. 